\title{
A case report of diffuse large B-cell thyroid lymphoma
}

\author{
Marwa Majdoub ${ }^{1}$, Ibtissem Ben Nacef $^{2}$, Sabrine Makni ${ }^{2}$, Mariem Ksantini $^{3}$, Nadia \\ Mchirgui $^{2}$, Imen Rojbi ${ }^{2}$, and karima khiari ${ }^{2}$ \\ ${ }^{1}$ Medical school of Sousse \\ ${ }^{2}$ Charles Nicolle Hospital \\ ${ }^{3}$ Hôpital Charles Nicolle
}

February 19, 2022

\begin{abstract} which accounts for 50 to $80 \%$ of thyroid primary lymphomas.

A case report of a diffuse large B-cell thyroid lymphoma

M.Majdoub ${ }^{1}$, I.Ben Nacef ${ }^{1}$, S.Makni ${ }^{1}$,M.Ksantini' ${ }^{2}$, N.Mchirgui ${ }^{1}$, I.Rojbi ${ }^{1}$, K.Khiari $^{1}$

\section{Others:}

Dr. Marwa Majdoub: majdoubmarwa14@gmail.com

Dr. Ibtissem Ben Nacef: bennacef.ibtissem@yahoo.com

Dr. Sabrine Makni: MakniSabrine@gmail.com

Dr. Mariem Ksantini : meriemksentini@yahoo.fr

Dr. Nadia Mchirgui : nadiamchirgui@yahoo.fr

Dr. Imen Rojbi: rojbiimen@yahoo.fr

Dr. karima khiari: karimakhiari@yahoo.fr
\end{abstract}

Primary thyroid lymphoma is rare. We describe the case of a 65-year-old woman with Hashimoto's thyroiditis who presented a 12-month history of neck swelling. Our investigation revealed the presence of a Diffuse Large B-Cell Lymphoma of the thyroid

1: Charles Nicolle Hospital, Endocrinology departement,Tunis, Tunisia

2: Charles NicolleHospital, Anatomopathology departement, Tunis, Tunisia

\section{Data availability statement}

The data that support the findings of this study are available from the corresponding author, upon reasonable request

\section{Financial sponsors, and support:}

There are no or special funding for the writing or publication of this paper. 


\section{Declaration of interest:}

\section{There are no conflicts of interest.}

\section{Patient consent:}

Written informed consent was obtained from the patient to publish this report in accordance with the journal's patient consent policy. The patients understand that their names and initials will not be published.

\section{Introduction:}

Thyroid lymphoma can be divided in two categories; primary (PTL) and secondary thyroid lymphoma (STL). STL is a part of a widespread neoplasia while PTL only involves , at diagnosis, the thyroid gland or the thyroid gland and local neck lymph nodes without contiguity or metastasis in other organs (1). PTL is a malignant transformation of normal lymphocytes in a normally non-lymphoid tissue (the thyroid).

It's a rare condition that only represents $5 \%$ of thyroid malignancies and $2.5 \%$ of lymphomas.

Here, we report the case of a 65 -year-old female with rapidly developing thyroid mass who represent the first thyroid lymphoma diagnosed in our department.

\section{Case presentation:}

We present the case of a 65-year-old woman with a known hypothyroidism due to Hashimoto's thyroiditis treated with levothyroxine $(1.12 \mathrm{mcg} / \mathrm{Kg} /$ day $)$.

She presented with a 12-month history of painless neck swelling. 3 months before consultation she reported the onset of a mild neck pain, an increase of the swelling's size and an occasional hoarseness.

The patient was vitally stable.

Physical examination showed a large nontender nodular neck mass moving with deglutition measuring approximatively $8 \mathrm{~cm}$, anterior cervical nontender adenopathies and a positive Pemberton's sign.

Cervical ultrasound revealed a left goiter; the left lobe measured $78 * 54 * 42 \mathrm{~mm}$, the right lobe $42 * 14 * 13 \mathrm{~mm}$, a $12 \mathrm{~mm}$ right nodule (EU-TIRADS 3), a $60 \mathrm{~mm}$ left thyroid hypoechoic lobulated nodule with microcalcifications (EU-TIRADS 5) and multiple anterior cervical adenopathies.

Fine needle aspiration was suggestive of a high-grade lymphoma.

Cervical lymph node and thyroid biopsies, shown in figure 1, confirmed the diagnosis of diffuse large B-cell thyroid lymphoma.

On immunohistochemical staining, the tumor cells were positive for CD20 as illustrated in figure 2, CD10, Bc16 and Ki67 (70\%).

These findings confirmed the diagnosis of diffuse large B-cell thyroid lymphoma arising in the setting of Hashimoto thyroiditis.

A computed tomography of the neck (Figure 3), Thorax, Abdomen and Pelvis was done for staging purpose, and it showed a large tissue mass of $5.2 \mathrm{~cm} \times 4.6 \mathrm{~cm} \times 8.9 \mathrm{~cm}$ size in the left lobe of the thyroid gland with capsular rupture in continuity with a group of left lymphadenopathies (channel III and IV), there was no evidence of any lesion elsewhere in the body.

No tumoral infiltration was found on the bone marrow biopsy.

The primary nature of this thyroid lymphoma was therefore established and it was classified IIE on the Ann arbor classification.

The blood counts, kidney and liver functions tests were within normal limits. Viral markers for hepatitis and human immunodeficiency virus were negative. Lactate dehydrogenase (LDH) was elevated (438 U/L). 
On the international Prognostic Index for Diffuse Large B-cell Lymphoma (IPI), the patient score was 2 indicating a Low-intermediate risk.

During her hospitalization in October 2020, the patient came in contact with a covid-19 patient and the polymerase chain reaction (PCR) test performed 5 days later was positive.

She remained asymptomatic during the 21 days of surveillance. Nevertheless, the decision was to delay the chemotherapy during her infection.

She then was started on an 8-cycle chemotherapy regimen R-CHOP: including cyclophosphamide (750 $\mathrm{mg} / \mathrm{m} 2)$, Adriamycin $(50 \mathrm{mg} / \mathrm{m} 2)$, vincristine $(2 \mathrm{mg} /$ day $)$ and prednisone $(60 \mathrm{mg})$ in association with rituximab $(375 \mathrm{mg} / \mathrm{m} 2)$.

Adjuvant radiotherapy is scheduled.

\section{Discussion :}

Similarly, to our case, PTL occurs usually in women after the sixth decade with a sex ratio of 2 to 8:1(2,3).

Thyroid lymphomas are mainly non Hodking lymphomas of B cell origin; the most common subtypes are diffuse large B-cell lymphoma (DLBCL) and mucosa-associated lymphoid tissue (MALT) lymphoma that account respectively for more than half of PTLs and $10 \%-30 \%$ of PTL. The coexistence of the two subtypes is possible suggesting the possibility of MALT transformation to DLBCL. $(2,4,5)$.

Autoimmune chronic lymphocytic thyroiditis is a well-established risk factor with a 40 to

80-fold increased risk of PTL. The prevalence of Hashimoto's thyroiditis ( HT) is deferent from one study to another based on the diagnosis criteria; overall the presence of antithyroperoxidase antibodies (ATPO) or clinical features of hypothyroidism was more common than a known medical history of HT(6). Many hypotheses aim to explain this association by the chronic antigenic stimulation, as well as the similarity in clonal bands between the PTL and HT patients(7, 8). However, according to Antonio Travaglino and al, the significant difference of HT's prevalence which is higher in MALT than DLBCL suggests different pathogeneses of these two lymphoma types(6).

In our clinical case, the diagnosis of HT was made on an elevated TSH and positive ATPO, shortly before the onset of the neck swelling. But the patient was never subjected to any thyroid function investigation, the thyroiditis might as well be asymptomatically evolving for years.

The most common presentations of PTL are a rapidly growing painless neck mass (few days to 36 months), symptoms due to compression such as dyspnea, dysphagia, coughing, hoarseness and even pain, in about $30 \%$ of the cases. It could also be revealed in $10 \%$ of cases by B-cell symptoms (fever, night sweats, or weight loss). DLBCL typically have a more rapid and aggressive presentation than MALT (2-4).

The case we report had a relatively long evolution of her thyroid mass, over 12 months. She reported the onset of pain and compression signs but not large B-cell symptoms. the Pemberton sign, witnessing the compression of major venous structures within a narrowed thoracic inlet against the enlarged thyroid, was positive.

Although it may be nonspecific, ultrasonography is a useful non-invasive way to explore thyroid nodes, and narrow down differential diagnosis. Hypoechogenicity, hypovascularity, lobulated shape and enhanced posterior echoes can suggest the diagnosis of lymphoma $(2,9)$.

Once malignancy is suspected upon clinical features or ultrasound findings, fine needle aspiration (FNA) seems as a less invasive tool for further characterization of the thyroid mass/nodule. Although the FNA's high sensitivity and specificity in investigating other types of thyroid cancer, it has a much lower accuracy when it comes to PTL; its sensitivity is below $56 \%$.

PTL frequently presents as an enlarged mass rather than a localized nodule. Distinguishing the PTL's histological aspect (MALT in particular) and that of chronic lymphatic thyroiditis, frequently associated, 
can be difficult; The riches of lymphoid tissue and a high proportion of intermediate centrocyte-like cells in low-grade PTL as compared to HT can only point to the PTL diagnosis $(2,9,10)$.

Appropriately, despite the improvement of FNA, biopsy is still necessary to confirm the diagnosis of PTL and define the histological subtype.

The advance in Immunohistochemistry has improved the diagnostic accuracy of cytology.

In our case's staining, the presence of CD20 indicates a B cell lineage to the lymphoid cells, however the positivity of CD10 in unusual in DLBCL(2).

PTL is staged according to the Ann Arbor classification; a locoregional disease corresponds to stages IE (extranodal involving only the thyroid gland) and IIE, while an advanced disease corresponds to stages IIIE (lymph nodes on both sides of the diaphragm) and IV (systemic dissemination). The disease is usually discovered at an early locoregional stage $(3,11)$, such as our patient who was staged IIE corresponding to the affection of the thyroid gland and regional lymph nodes above the diaphragm only.

Cancer patients seem to be more susceptible to SARS-CoV-2 infection along with a higher risk of complications. This can be explained by these patients' advanced age, comorbidities, high prevalence of smoking, their weakened immune system by the malignancy itself and/or its treatment $(12,13)$.

In this case report, our patient had no medical history of immunodepression or comorbidities, her tests for hepatitis and human immunodeficiency virus were negative.

Necessary measures to reduce of nosocomial SARS-CoV-2 acquisition were adopted during her hospitalization. Nevertheless, she was diagnosed with covid-19; she did not present any symptoms nor complications.

Record of patients who underwent chemotherapy or surgery in the month preceding their covid-19 infection having a numerically higher risk of clinically severe events compared to those who did not receive chemotherapy or surgery(14), contributed to our decision to delay the patient's chemotherapy.

The rareness of PTL makes establishing a well-defined therapy approach quite challenging. Most others report better outcome and disease control with combined chemoradiation therapy even in early-stage DLBCL. Commonly, PTL responds to a multi-agent chemotherapy including cyclophosphamide, doxorubicin, vincristine, and prednisone (CHOP) (9, 15-19). The association of Rituximab, a monoclonal B-cell antibody that binds to the CD20 antigen, in elderly patient has been shown to be of benefit in improving event-free and over-all survival $(19,20)$.

Nevertheless, the outcome of this disease does not only depend on treatment modality; poor prognosis is related to histological subtype, as DLBCL is more aggressive, advanced stage, age above 60 years, rapid tumor growth, presence of local obstructive symptoms or B symptoms, mediastinal involvement, elevated $\operatorname{LDH}(5,17,21)$.

\section{Conclusion :}

Primary thyroid lymphoma is both a rare thyroid malignancy and a rare lymphoma type.

It's not yet easily diagnosed despite the improvement of imaging techniques and FNA.

Due to the rarity of this affection, no unanimous treatment guideline is currently available. Every case should therefore be treated individually by a multidisciplinary team.

\section{Author contribution:}

All authors contributed to the content of this manuscript; K.KHIARI, I.ROJBI, N.MCHIRGUI, Y.LAKHOUA reviewed the manuscript. M.KSANTINI, I.BENNACEF and M.MAJDOUB collected the data for the case report. M.MAJDOUB wrote the paper with input from all authors.

\section{References:}


1. Ansell SM, Grant CS, Habermann TM. Primary thyroid lymphoma. Seminars in oncology. 1999;26(3):31623.

2. Stein SA, Wartofsky L. Primary thyroid lymphoma: a clinical review. J Clin Endocrinol Metab. 2013;98(8):3131-8.

3. Derringer GA, Thompson LD, Frommelt RA, Bijwaard KE, Heffess CS, Abbondanzo SL. Malignant lymphoma of the thyroid gland: a clinicopathologic study of 108 cases. Am J Surg Pathol. 2000;24(5):62339.

4. Widder S, Pasieka JL. Primary thyroid lymphomas. Curr Treat Options Oncol. 2004;5(4):307-13.

5. Graff-Baker A, Roman SA, Thomas DC, Udelsman R, Sosa JA. Prognosis of primary thyroid lymphoma: demographic, clinical, and pathologic predictors of survival in 1,408 cases. Surgery. 2009;146(6):1105-15.

6. Travaglino A, Pace M, Varricchio S, Insabato L, Giordano C, Picardi M, et al. Hashimoto Thyroiditis in Primary Thyroid Non-Hodgkin Lymphoma. Am J Clin Pathol. 2020;153(2):156-64.

7. Kossev P, Livolsi V. Lymphoid lesions of the thyroid: review in light of the revised European-American lymphoma classification and upcoming World Health Organization classification. Thyroid : official journal of the American Thyroid Association. 1999;9(12):1273-80.

8. Moshynska OV, Saxena A. Clonal relationship between Hashimoto thyroiditis and thyroid lymphoma. J Clin Pathol. 2008;61(4):438-44.

9. Sakorafas GH, Kokkoris P, Farley DR. Primary thyroid lymphoma (correction of lympoma): diagnostic and therapeutic dilemmas. Surg Oncol. 2010;19(4):e124-9.

10. Zhang L, Castellana M, Virili C, Crescenzi A, Giorgino F, Zucca E, et al. Fine-needle aspiration to diagnose primary thyroid lymphomas: a systematic review and meta-analysis. Eur J Endocrinol. 2019;180(3):177-87.

11. Graff-Baker A, Sosa JA, Roman SA. Primary thyroid lymphoma: a review of recent developments in diagnosis and histology-driven treatment. Curr Opin Oncol. 2010;22(1):17-22.

12. Moujaess E, Kourie HR, Ghosn M. Cancer patients and research during COVID-19 pandemic: A systematic review of current evidence. Crit Rev Oncol Hematol. 2020;150:102972.

13. Weinkove R, McQuilten ZK, Adler J, Agar MR, Blyth E, Cheng AC, et al. Managing haematology and oncology patients during the COVID-19 pandemic: interim consensus guidance. Med J Aust. 2020;212(10):481-9.

14. Liang W, Guan W, Chen R, Wang W, Li J, Xu K, et al. Cancer patients in SARS-CoV-2 infection: a nationwide analysis in China. Lancet Oncol. 2020;21(3):335-7.

15. Doria R, Jekel JF, Cooper DL. Thyroid lymphoma. The case for combined modality therapy. Cancer. 1994;73(1):200-6.

16. Mian M, Gaidano G, Conconi A, Tsang R, Gospodarowicz MK, Rambaldi A, et al. High response rate and improvement of long-term survival with combined treatment modalities in patients with poor-risk primary thyroid diffuse large B-cell lymphoma: an International Extranodal Lymphoma Study Group and Intergruppo Italiano Linfomi study. Leuk Lymphoma. 2011;52(5):823-32.

17. Onal C, Li YX, Miller RC, Poortmans P, Constantinou N, Weber DC, et al. Treatment results and prognostic factors in primary thyroid lymphoma patients: a rare cancer network study. Ann Oncol. 2011;22(1):156-64.

18. Peixoto R, Correia Pinto J, Soares V, Koch P, Taveira Gomes A. Primary thyroid lymphoma: A case report and review of the literature. Ann Med Surg (Lond). 2017;13:29-33. 
19. Mack LA, Pasieka JL. An evidence-based approach to the treatment of thyroid lymphoma. World J Surg. 2007;31(5):978-86.

20. Pfreundschuh M, Schubert J, Ziepert M, Schmits R, Mohren M, Lengfelder E, et al. Six versus eight cycles of bi-weekly CHOP-14 with or without rituximab in elderly patients with aggressive CD20+ B-cell lymphomas: a randomised controlled trial (RICOVER-60). Lancet Oncol. 2008;9(2):105-16.

21. Tupchong L, Hughes F, Harmer CL. Primary lymphoma of the thyroid: clinical features, prognostic factors, and results of treatment. Int J Radiat Oncol Biol Phys. 1986;12(10):1813-21.

\section{Hosted file}

figure_article.docx available at https://authorea.com/users/461372/articles/557079-a-casereport-of-diffuse-large-b-cell-thyroid-lymphoma 